\title{
Study on 3D-Micro discretization algorithm of 3D printing
}

\author{
Xuepeng Liu', ${ }^{1, a}$ Dongmei Zhao ${ }^{1, b}$ \\ ${ }^{1}$ Zhongshan Polytechnic, P.R. China \\ aliuxuepeng1026@yahoo.com.cn, bdzmeihn@163.com
}

Keywords: 3D printing, octree, Similar Figure Topological rule, Minimum Distance rule

\begin{abstract}
The matching points relation of stereo-picture is built by adjacent slices through setting up mark for matching points conforming to three rules: Minimum Distance rule, evenly--Distributed rule and the Similar Figure Topological rule through reconstructing surface of microstructure. Based on the traditional ray tracing, 3D-Micro discretization algorithm is accomplished by octree tracing face clustering algorithm to build micro-constructre surface. Finally the 3d-picture is drawn for a theoretical basis of 3D printing.
\end{abstract}

\section{Introduction}

In the design of software system in rapid prototyping and slice processing is one of the key content. This article discussed the $3 \mathrm{~d}$ model discretization algorithm slice processing. By slice processing, the complex three-dimensional model into planar contour information, the computer according to its contour information layer by layer building objects. The contour information accuracy directly affect the effect of molding. Moreover, with the development of $3 \mathrm{~d}$ printing, print the target object is bigger and bigger, more and more complex, which often result in processing takes big slice. And through the optimization algorithm, can significantly reduce the consumption of time on file pre-processing, and can improve the effect of printing.

Rendering $3 \mathrm{~d}$ graphics on computer, you must set up three-dimensional structure of dot, line and surface data structure, and determine the corresponding relation, the adjacent relation, finally through a series of coordinate transformation, the graph blanking processing, draw on the computer screen is stereo $3 \mathrm{~d}$ graphics. As in the series of parallel sections of this procedure is for the two-dimensional plane figure on the topology structure, on every plane of all points with the same $\mathrm{z}$ coordinate values, so in each layer in the plane by a uniform variable $\mathrm{z}$ coordinates, then use a three-dimensional array figure is enough to represent all points of $\mathrm{x}, \mathrm{Y}$ coordinate values and the corresponding serial number value. In each layer is established after the data structure of points in the plane, set point of the corresponding relationship between adjacent plane is the key to establish the data structure, due to the level in the current section reconstruction is still not fully resolved in some problems, such as contour correspondence, bifurcate of profile stitching, key issues, and the microstructure of materials such as general with continuous entity, such as grain structure, therefore, this thesis mainly research

In establishing a series of serial section adjacent cross section of a graphic of a single corresponding points matching relationship between contour lines.

\section{The Discretization Strategy}

Points matching relationship in the process, must establish the connection between the two match points. As long as a point in the cross section of adjacent with matching point, the program established after points matching relationship between adjacent sections, can find the corresponding matching points. To do this, set a flag values for each point, the value of adjacent section in the serial number of the matching points. Program in the realization of the adjacent two section points matching, automatically gives two points marked values to the corresponding serial number of the matching point, finally outputs the matching point pairs. In order to keep the order of the input point of orderly and conducive to the program automatically match correctly, should be in the same direction (clockwise or counterclockwise) as a cross section contour point number and input, and as far as 
possible from the same place in different cross section as a starting point to form closed graph point number.

\subsection{Distance Preference.}

If the two basic overlap, the center of the closed graph and outer contour shape is basically similar, the shortest distance between two points is two points for matching the two points of each other, this is the priority principle of distance. Minimum distance principle suitable for axisymmetric shape microstructure, such as rod, cone, sphere, etc., and can realize the program automatically determine the matching points. If I and $\mathrm{j}$ plane of two adjacent plane respectively, $\mathrm{xi}, \mathrm{yi}, \mathrm{xj}, \mathrm{yj}$, respectively the I and $\mathrm{j}$ plane coordinates $\mathrm{X}$ and $\mathrm{Y}$ coordinates, when the distance between two points, $\mathrm{d} 2=(\mathrm{xi}-\mathrm{xj}) 2+$ (yi - yj) 2 hours, both for mutual matching points.

\subsection{Structure Similarity Principle.}

In the design application, can make the program automatically determine points matching relationship of course is the best. But when graphic structure is large, graphics without overlap between center, had better use artificial method to intervene. Design program to judge whether the structure of the two graphics similar it is difficult, so according to the structure similarity using artificial method of graphical predetermined some mutual matching points, then computer is used to determine whether the rest of the points matching relations, this is the graph topology similarity principle. Using artificial method to determine in advance some mutual matching points, to avoid the large difference in graphic topology with a minimum distance principle point caused by the unreasonable matching, up to twice the result with half the effort. Manual to determine the point match each other, is according to the topology of the graph, find out the location and shape of the most close to the two points, put their flag values the same integer value. Determined in the following process points matching relations, will be automatically detected with the same id value point, and put these points identified as mutual matching points, with a minimum distance principle to determine the rest of the points matching relationship.

\subsection{Percentage Distribution Principle.}

When the program automatically determine points matching relationship between adjacent sections, if to be determined in two cross-section is large, the number of points, according to the relationship between the proportion of the number of points, automatically determine the number of points less in the cross section point should be another section of the number of matching points and the area, and then in accordance with the principle of minimum distance in this area to determine the point matching relations. Uniform distribution points matching more reasonable, careful.

Program when determining points matching relationship, if the shape of the adjacent section is similar, neither the matching points, predetermined program is two section of the number of points, in the case of the number of points were similar, with the minimum distance principle to determine the point matching relationship, if the number of points is large, will calculate some proportion relations, principles and the principle of minimum distance with uniform distribution points matching relations; If adjacent cross section graph topology is not similar, both the predetermined matching points, program will, in turn, find out the two adjacent to the predetermined matching points points, with a minimum distance principle and the principle of uniform distribution determine points matching relationship between the two points. in

Determine all the points, the matching relation between the adjacent section of matching points stored in the corresponding data structure.

In rendering $3 \mathrm{~d}$ graphics data, the point is the most basic data. After the organization better data, therefore, to determine the good points matching relationship between adjacent sections, to the side of the drawing materials microstructure stereoscopic structure laid the foundation, this also is one of the most critical step. In establishing a little later, the matching relation between is ready to set up $3 \mathrm{~d}$ graphic data structure basic data, and also to organize the three-dimensional graphics data structure of line and plane laid a foundation. 


\section{Octree Clustering Mesh Simplification Algorithm}

The idea is to convert the triangular mesh of the object model to a big figure, corresponding to the peak of triangle into the method of vector line Angle between adjacent triangles as connection weights of figure above, the short circuit on the tree root node and other nodes between weights and defined as the distance between the corresponding triangular, less than a given threshold of the triangle and the root node belong to the same class. Initial stage all triangles with belong to the category, freely choose a triangle"

As the center, calculation of the distance between a and other each triangle, then find out the maximum distance triangle to form a new class, repeat the previous operations, until you get the number of classes to fulfill the requirements of the user to specify. By plane in approximate plane triangle in the grid clustering together for a class, then the triangle of the same kind of edge features and feature point extraction, formed a regional, again to limited area of the triangle subdivision. So as to achieve the goal of mesh simplification. We noticed that the main cost calculation on the division of the algorithm is also face clustering, obviously, when the three dimensional model is very complex, contains too many triangles, calculating the distance form the centre of all kind of the triangle to the rest of the all will be very time consuming, it is for the application of real-time demand higher unbearable. Therefore, we propose an improved based on space division to triangle, then, only mutually adjacent triangles are likely in the approximation of the plane. So we put the triangle of clustering, thereby reducing calculation, improve the simplified speed. The algorithm using octree to adaptive $3 \mathrm{~d}$ object space division component accelerated in the presence of clustering mesh simplification algorithm. We already know that the basic idea of clustering is put in approximate plane

\section{Summary}

With the development of 3 d printing technology and business applications, "mass customization" will become the important production mode. $3 \mathrm{~d}$ printing and the close combination of modern service industry, to subdivide spawned new industries and new business model, create a new economic growth point. $3 \mathrm{~d}$ printing technology development of product technology, manufacturing technology and management technology make the enterprise have the ability of rapid response to market demand [1-4], especially form rich variety of customers to adapt to the global market, to realize the remote custom, different design, local production and sales of harmonization new production mode, production model, business model, and many other aspects of fundamental changes

\section{References}

[1]Yang S F, Julian R. A dry powder jet printer for dispensing and combinatorial research [J] . Powder Technology, 2004 ( 142 ) :219 -222.

[2] Blazdell J. Application of a continuous ink jet printer to solid freeforming of ceramics

[J] . Journal of Materials ProcessingTechnology, 2000( 99) : 94-102.

[3] Robert Q. Stuart M, Phil R, et al. Shaping our national competencyin manufacturing [EB/OL] . [2012 - 09- 18]. http: / /www. materialsktn. net /.

[4] Terry W. Additive manufacturing and 3D printing state of the industry. Annual Worldwide Progress R eport Wohlers Associstions, 2012. 